\title{
IDENTIFYING NEAR-IR VARIABLE Be STARS
}

\author{
S.M. DOUGHERTY \\ John Moores University, Liverpool, England \\ and \\ A.R. TAYLOR \\ University of Calgary, Calgary, Canada
}

\begin{abstract}
Near-IR variable Be stars are identified from multi-epoch observations spanning 20 years using a statistical technique. In this manner, observations from different observing sites can be meaningfully combined and compared. A more thorough investigation of the incidence and properties of IR variability in Be stars as a class of stars is then possible.
\end{abstract}

Although IR variations on time scales ranging from months to years have been observed in Be stars, the statistics and properties of the IR variations in the few studies undertaken differ dramatically. To date there has been no systematic search for variations at near-IR wavelengths from a large sample of Be stars. A large sample of IR observations over a number of epochs allows better statistics of the long term variations of Be stars to be determined than in previous analyses. Furthermore, with a large sample of stars it is possible to use the sample itself to correct for systematic differences between epochs.

The data set consists of magnitudes from several epochs of observation, both new observations and those taken from the literature (Dougherty and Taylor, 1994). Observation of the $i$ th star at the $j$ th epoch yields a magnitude $m_{i j} \pm \sigma_{i j}, \sigma_{i j}$ being the $1 \sigma$ uncertainty in the magnitude. Consider the distribution of deviations $\Delta_{i j}$, of the magnitudes $m_{i j}$ from the mean magnitude of the $i$ th star, $\bar{m}_{i}$ i.e.

$$
\Delta_{i j}=m_{i j}-\bar{m}_{i} .
$$

To identify stars that have variable magnitudes, a sample of non-variable stars was first determined. The deviations for the non-variables will be mainly confined within $\pm 3 \sigma$ of the mean of the distributions of $\Delta_{i j}$. An initial sample of non-variables was defined as those stars for which the deviations satisfy $\left|\Delta_{i j}\right| \leq 2.5 \sigma_{\Delta_{i j}}$.

Once the non-variable stars were identified, the distribution of deviations for non-variables at each individual epoch were corrected so that a nonvariable star has, on average, the same magnitude at all epochs, and that the width of the distributions $\Delta_{i j}$ of non-variable stars are consistent with the quoted rms uncertainties in the magnitudes $m_{i j}$. Assuming the uncertainties are uniform at epoch $j$, then the distribution of $\Delta_{i j} / \sigma_{i j}$ for non-variable stars at epoch $j$ will have a standard deviation of unity.

Stars for which $\left|\Delta_{i j}\right|>4 \sigma_{i j}$ at any passband were identified as IR variables. The possibly variable stars were defined using the deviation that 
TABLE I

Summary of number of variable stars

\begin{tabular}{ccccc}
\hline & $\mathrm{J}$ & $\mathrm{H}$ & $\mathrm{K}$ & $\mathrm{L}$ \\
\hline \# of stars with $\Delta / \sigma>4$ & 10 & 15 & 21 & 13 \\
Total \# of stars & 112 & 116 & 117 & 89 \\
$\%$ & 8.9 & 12.9 & 17.9 & 14.6 \\
\hline
\end{tabular}

defines a sample in which there will be, on average, one non-variable event. This corresponds to a deviation of $3.2 \sigma$ for this data sample. Hence, stars with $3.2 \sigma_{i j}<\left|\Delta_{i j}\right|<4 \sigma_{i j}$ were identified as possible IR variables.

In this manner, the variable Be stars are: $o$ Cas, BK Cam, $120 \mathrm{Tau}, \omega$ Ori, HR 2309, FV CMa, $\omega$ CMa, FW CMa, HR 2921, HR 3858, HR 4930, $\mu$ Cen, V767 Cen, $\chi$ Oph, 66 Oph, V3031 Sgr, V4024 Sgr, V923 Aql, HR 7983 $59 \mathrm{Cyg}, 31 \mathrm{Peg}, \pi$ Aqr, $8 \mathrm{Lac}, \epsilon$ PsA, EW Lac, $\beta$ Psc. The following stars are possible IR variables: $\gamma$ Cas, $\psi$ Ori, $\zeta$ Tau, HR 3135, $\kappa$ Dra, $\eta$ Cen, 48 Lib.

A more complete version of this analysis can be found in Dougherty and Taylor (1994) along with a discussion of the properties of the variations.

\section{References}

Coté, J. and Waters, L.B.F.M., 1987, Astronomy and Astrophysics, 176,93

Dougherty, S.M., Waters, L.B.F.M., Burki, G., Coté, J., Cramer, N., van Kerkwijk, M.H., and Taylor, A.R., 1994, Astronomy and Astrophysics, submitted.

Dougherty, S.M., and Taylor, A.R., 1994,Monthly Notices of the RAS, submitted. 